\title{
Improving the Quality of Early Hearing Detection and Intervention Services through Physician Outreach
}

\author{
Karen Munoz, Ed.D., ${ }^{1}$ Lenore Shisler, M.S., ${ }^{2}$ Mary Pat Moeller, Ph.D., ${ }^{3}$ \\ and Karl R. White, Ph.D. ${ }^{4}$
}

\section{ABSTRACT}

Physicians and other health-care providers should play a central role in helping families of children with permanent hearing loss receive timely and appropriate screening, diagnostic, and early intervention services. Because the technology and procedures for ensuring timely and appropriate services for infants and young children with hearing loss have changed so dramatically over the past 15 years, many health-care providers are not well informed about the best ways to provide effective services. Audiologists can help to ensure that physicians and other health-care providers are better informed about the services needed by infants and young children with hearing loss. This article notes some of the areas where information and support is most needed and suggests resources and strategies for addressing these needs.

KEYWORDS: Hearing loss, childhood, physician, continuing education

Learning Objectives: As a result of this activity, the participant will be able to (1) describe the physician role in supporting follow-up from newborn hearing screening, (2) identify strategies for communicating key information to physicians, and (3) identify resources to support timely follow-up from newborn hearing screening.

The importance of early identification and management of childhood hearing loss is well recognized. During the past decade, the United States has experienced a dramatic in-
'Assistant Professor of Communicative Disorders and Deaf Education, Deputy Director, National Center for Hearing Assessment and Management, Utah State University, Logan, Utah; ${ }^{2}$ National Center for Hearing Assessment and Management, Utah State University, Logan, Utah; ${ }^{3}$ Director, Center for Childhood Denfness, Lied Learning and Technology Center, Boys Town National Research Hospital, Omaha, Nebraska; ${ }^{4}$ Professor of Psychology, Director, National Center for Hearing Assessment and Management, Utah State University, Logan, Utah.
Address for correspondence and reprint requests: Karen Munoz, Ed.D, National Center for Hearing Assessment and Management, Utah State University, 2880 Old Main Hill, Logan, UT 84322 (e-mail: Karen.Munoz@usu.edu).

Physician Outreach for Hearing Health Care: Contemporary Issues; Guest Editor, Carole E. Johnson, Ph.D., Au.D.

Semin Hear 2009;30:184-192. Copyright (0) 2009 by Thieme Medical Publishers, Inc., 333 Seventh Avenue, New York, NY 10001, USA. Tel: +1(212) 584-4662. DOI 10.1055/s-0029-1225403. ISSN 0734-0451. 
crease in the number of newborns being screened for hearing loss. Currently, $>95 \%$ of infants are screened before hospital discharge, compared with $<20 \%$ in $1997 .^{1}$ Hearing screening in infancy also has become the medicolegal standard of care with 42 states now having statutes related to universal newborn hearing screening. ${ }^{2,3}$ Additionally, early hearing detection and intervention (EHDI) programs have been implemented in each U.S. state and territory with the goal that all newborns will be screened for hearing loss and infants not passing the screening will receive timely and appropriate audiological evaluation and early intervention services. ${ }^{4}$

Although remarkable advances in newborn screening have been achieved, linking families to vital follow-up services after a failed hearing screen remains challenging. Unfortunately, analysis of recent state EHDI data reported to the Centers for Disease Control and Prevention revealed that among the $2.2 \%$ of infants referred for follow-up after newborn screening, only $38.7 \%$ were documented as having received a diagnostic evaluation. ${ }^{5}$ Although some of the infants for whom receipt of a diagnostic evaluation could not be documented might have been evaluated and not reported, these data provide strong evidence that many infants who fail newborn hearing screening are not receiving services.

Undetected and untreated hearing loss negatively affects children's speech, language, cognitive, and social-emotional development, as well as later academic performance. ${ }^{6-8}$ Newborn hearing screening helps prevent or minimize such delays only if appropriate and comprehensive follow-up services are obtained in a timely manner.

Primary health-care providers, in partnership with other professionals, play a pivotal role in creating a medical home to ensure that children referred from newborn hearing screening receive the needed follow-up care. As defined by the American Academy of Pediatrics (AAP), the ideal medical home should be accessible, family centered, continuous, comprehensive, coordinated, compassionate, and culturally effective.' In the medical home model, the physician is a case manager responsible for the ongoing care of the child. Even though congenital hearing loss occurs more frequently than any other birth defect, it is still a relatively low-incidence condition, affecting $\sim 3$ per 1000 newborns. ${ }^{10}$ Thus most individual physicians will serve only a small number of infants with hearing loss during his or her career. Added to that, the field of early hearing detection and intervention has undergone dramatic changes over the past two decades. This has made it difficult for physicians and other health-care providers to stay up to date regarding how to provide the best services for infants and young children with permanent hearing loss. Physicians will therefore require support from other knowledgeable professionals as they assist families through the multistep diagnostic and intervention process. Audiologists are in an excellent position to provide the information and support needed by health-care providers in this area.

Audiologists, speech-language pathologists, professional associations, and state EHDI coordinators who understand the multistep screening, diagnosis, and intervention processes, as well as the points at which families are at risk for becoming lost to follow-up, need to find ways to cooperate with primary care physicians to share critical information and resources. Outreach efforts will be most effective if participants understand physicians' perspectives about EHDI and knowledge gaps commonly affecting their ability to serve as the medical home for children who have a suspected or confirmed hearing loss. This article provides an overview of these issues and summarizes outreach strategies and national and state resources that can be used to promote effective physician involvement in EHDI programs.

\section{PHYSICIANS' ATTITUDES, PRACTICES, AND KNOWLEDGE}

Primary care physicians will best be able to support families of infants and young children with suspected or confirmed hearing loss if they understand the critical components of hearing screening, diagnosis, and intervention and the time-sensitive nature of these services. The 2007 Joint Committee on Infant Hearing (JCIH) Position Statement ${ }^{11}$ provides valuable 
direction to professionals involved in each stage of the EHDI process including (1) screening before 1 month of age, (2) diagnostic evaluation before 3 months of age, (3) fitting of amplification within 1 month of diagnosis, and (4) enrollment in an appropriate early intervention program as soon as possible after diagnosis, but no later than 6 months of age. To provide appropriate support to primary health-care providers, audiologists and related professionals must understand what physicians do and do not know related to each of these service elements.

Critical gaps in physicians' attitudes, practices, and knowledge about early hearing detection and intervention were recently documented in focus group findings ${ }^{12}$ and through a national survey to which 1968 physicians responded. ${ }^{13}$ Results of the survey (referred to hereafter as the "Physician Survey") are presented in the following sections as examples of areas in which primary health-care providers need more education and support if they are to be effective partners in providing high-quality care for infants and young children with permanent hearing loss.

\section{Newborn Screening and Referral for Audiological Assessment}

Families are particularly at risk for becoming lost to follow-up after referral from newborn hearing screening and before audiological evaluation. When an infant fails the newborn hearing screening, the infant's primary healthcare provider should always be notified about the need for follow-up screening or audiological testing. In a recent survey of state EHDI program coordinators, ${ }^{14}$ only $73 \%$ of the coordinators reported that this happens "almost all of the time" (defined in the survey as happening for 80 to $100 \%$ of the referred children). These data suggest that notification of the primary health-care provider is not happening in many cases. Even when there is an intent to notify the primary health-care provider, it may not occur for various reasons: The physician of record at the hospital may not be the one who actually provides care for the infant, parents may not continue with the same physician who cared for the infant in the hospital, or families may access a birthing hospital in a nearby state rather than their home state, thereby complicating the communication of results from the state EHDI office to the physician in their home state. Only $12 \%$ of pediatricians and $17 \%$ of family physicians in the Physician Survey responded that they receive screening results on $>50 \%$ of their patients. ${ }^{13,15}$ Unfortunately, physicians often assume that no news is good news as it relates to test results. This is not a safe assumption because breakdowns in communication are common.

Some hospitals follow a one-stage protocol (screening completed before discharge); others use a two-stage protocol (newborns not passing the screening before discharge return for an outpatient screening). In either case, the screening process should be completed before 1 month of age. Repeating the screening process multiple times is not advised. Instead, infants who fail the complete newborn hearing screening process should be promptly referred for a diagnostic audiological evaluation as soon as possible and always before 3 months of age. Physicians need to know what should be included in a comprehensive audiological evaluation for an infant, ${ }^{16}$ and they need a list of qualified pediatric audiologists in the area who have the equipment, experience, and expertise to complete such evaluations in a timely manner. The current shortage of pediatric audiologists means that the availability of audiological services for infants may be limited and wait lists long, particularly in rural locations. ${ }^{11}$

\section{Referral for Medical Evaluation}

Once an audiological diagnosis of a hearing loss has been made, the referral and case management process becomes more complex and the physician's need for information increases. Only $14 \%$ of the respondents in the Physician Survey reported that their training program had adequately prepared them to meet the needs of infants with permanent hearing loss. ${ }^{13}$ When an infant is diagnosed with a hearing loss, the primary health-care provider should immediately begin coordinating referrals to a team of professionals who must work together to identify the etiology of 
the hearing loss and make recommendations for medical, audiological, and educational follow-up. According to the JCIH, infants and toddlers with permanent hearing loss should be referred to an otolaryngologist with specific knowledge of pediatric hearing who can provide medical clearance for amplification, as well as to a geneticist and an opthalmologist. ${ }^{11}$ Many physicians will need guidance from audiologists to make appropriate referrals to otolaryngology and other medical specialists. For example, in the Physician Survey the need for a referral to a geneticist was recognized by only $11 \%$ of pediatricians, $3 \%$ of family practitioners, and $22 \%$ of otolaryngologists. Furthermore, the need to refer to an ophthalmologist was recognized by only $1 \%$ of pediatricians, $0 \%$ of family practitioners, and $7 \%$ of otolaryngologists. ${ }^{13,15}$

\section{Amplification}

The role of the physician is critical to ensure that infants and young children diagnosed with permanent hearing loss have the opportunity to be fitted with hearing aids within 1 month of diagnosis. ${ }^{11,17}$ Unfortunately, $53 \%$ of the respondents to the Physician Survey did not know that infants who were $\leq 3$ months of age could be fit with hearing aids, and $18 \%$ thought it necessary to wait until the child was $\geq 12$ months of age. ${ }^{13}$ In cases where hearing loss is so severe that hearing aids cannot provide sufficient amplification for the acquisition of spoken language, physicians should be prepared to discuss information about cochlear implants with families. ${ }^{17}$ Almost $50 \%$ of respondents to the Physician Survey were incorrect about the type and level of hearing loss that would make a child an appropriate candidate for a cochlear implant, and almost $70 \%$ reported a lack of confidence in discussing this surgical procedure with families of such children. ${ }^{13,15}$ This points out the need for physicians not only to understand what constitutes current evidence-based practice, but also their need for access to family-friendly information that can be used in discussing a child's hearing loss with family members. The Web site www.babyhearing.org is an excellent source of such information. ${ }^{18}$

\section{Referral for Early Intervention} and Family Support Services

As soon as a permanent hearing loss is diagnosed, federal law (the Individuals with Disabilities Education Act) requires that the child be referred to the state's early intervention program. ${ }^{19}$ However, in a recent survey of state EHDI program coordinators, ${ }^{14}$ only $47 \%$ responded that such referrals happen "Almost Always" (defined in the survey as being for 80 to $100 \%$ of the children diagnosed with hearing loss). The child's primary health-care provider is in an ideal position to ensure that such referrals are made promptly and that parents understand the services that are available at little or no cost to them and their child.

Throughout the early intervention (EI) process, the physician is in a position to support the family as they monitor progress and make decisions about the services they want their child to receive. The physician can serve as a sounding board if the child is making slower progress than expected, refer for specialty evaluations, and be involved in differential diagnosis of additional disabilities. For physicians to collaborate effectively, they need to know how and where to refer families, have easy access to family-friendly information, understand what services $\mathrm{EI}$ is providing, and receive and read reports from EI providers. In essence, physicians need to be educated consumers of EI services.

Physicians also need information on specific eligibility criteria for infants diagnosed with hearing loss in their state and an understanding of the role of the early intervention team. This information is available for each state at www.infanthearing.org. ${ }^{20}$ Physicians also should understand that speech-languagepathologists, trained in aural habilitation, are in a key position to help families who have children with permanent hearing loss. However, in the Physician Survey, the need for a referral to a speech-language pathologist was recognized by only $29 \%$ of pediatricians, $13 \%$ of family practitioners, and $14 \%$ of otolaryngologists. ${ }^{13,15}$

Another important aspect of early intervention involves providing information and education to families about communication strategies for their child. Deciding whether to communicate using sign language, cued speech, 
or spoken language is often a very difficult decision for families; consequently, many parents turn to physicians for input. Because the selection of a communication methodology for the child is a complex and ongoing process, physicians need to have a core understanding of the issues and know where to direct families for complete and unbiased guidance. However in the Physician Survey, 57\% of respondents said they were "Not Confident" in talking with parents about the "use of sign language versus auditory/oral communication."

In the Physician Survey, respondents also expressed the need for training on how to better counsel parents when their infant is diagnosed with hearing loss. A common sentiment expressed in the focus group was "How worried should I tell parents they need to be about this?"13 The www.babyhearing.org Web site provides materials that health-care providers can use when talking with families about a child's hearing loss. ${ }^{18}$ Table 1 lists additional suggestions.

\section{Ongoing Surveillance during Early Childhood}

For children born at home or not screened at birth for other reasons, physicians need to know where early childhood hearing screening is offered within the community. Such screening is particularly important for children who exhibit one of the risk indicators for hearing loss identified by the JCIH. ${ }^{11} \mathrm{Un}_{\mathrm{n}}$ fortunately, the results of the Physician Survey show that many physicians do not know the conditions that put an infant or young child at risk for hearing loss. ${ }^{13}$ Also, because

Table 1 Suggestions for Meeting Information Needs of Physicians Related to Early Identification and Treatment of Permanent Hearing Loss

Overview of EHDI Process

- Disseminate AAP Universal Newborn Hearing Screening Guidelines for Pediatric Medical Home Providers. ${ }^{27}$

- Distill and disseminate relevant guidance from JCIH 2007 Statement. ${ }^{11}$

Newborn Screening and Referral for Audiological Assessment

- Encourage physicians to obtain hearing screening results by contacting birth hospitals or their state EHDI program. ${ }^{29}$

- Encourage physicians to modify standard history forms to ask parents the results of newborn hearing screening and follow-up testing.

- Reference evidence-based diagnostic protocols. ${ }^{16}$

- Provide list of local pediatric audiologists who have the equipment, expertise, and experience to conduct a comprehensive diagnostic evaluation.

Referral for Medical Evaluation

- Provide list of local otolaryngologists who have expertise with infants.

- Supply information about need for, and importance of, referral to medical specialists. ${ }^{11}$

- Provide list of local medical specialists, including geneticists and ophthalmologists who have expertise with infants.

Amplification

- Provide list of local pediatric audiologists who can fit hearing aids.

- Provide list of pediatric cochlear implant teams in the area.

- Reference evidence-based amplification protocois. ${ }^{17}$

Referral for Early Intervention and Family Support

- Provide list of local early interventionists and speech-language pathologists skilled in working with infants with hearing loss.

- Disseminate parent-friendly materials for physicians to use in counseling and supporting families. ${ }^{18}$

Ongoing Surveillance

- Provide list of local resources where infants/toddlers can receive hearing screening.

- Provide training materials and support to physicians who want to do early childhood screening as a part of well-child visits. ${ }^{30,31}$ 
hearing loss can occur at any time, ongoing surveillance of hearing problems is needed for all children. For infants who pass newborn hearing screening, the JCIH states that physicians should be providing regular surveillance during periodic well-child visits including "providing an objective standardized screening of global development with a validated screening tool at 9,18 , and 24 to 30 months of age or anytime if the healthcare professional or family has a concern." 11 If an infant does not pass the speech-language portion of the global screening, the JCIH recommends that referral be made for both an audiological and speech-language evaluation. It is important that audiologists work with physicians and other health-care providers to implement this important aspect of early hearing detection.

\section{EDUCATIONAL OUTREACH CONSIDERATIONS}

What physicians need to know changes as children in their care move through the continuum of EHDI services. Given the breadth of information needs related to hearing loss, it is noteworthy that physicians stated during the focus groups that it is not realistic for them to become "experts. ${ }^{n 2}$ Instead, they would like to know where to access evidence-based practice guidelines. Audiologists can assist in this process by distilling information from various sources into practical "next steps" that can be conveyed to physicians in an appropriate format. In communicating information, five primary themes emerged from the focus group work with physicians ${ }^{12}$ that are especially relevant for audiologists and other professionals to understand:

1. Recognition of the time constraints that physicians face in their daily practice and the limited frequency with which they encounter permanent hearing loss among infants and young children.

2. Information provided to primary healthcare providers does not need to contain detailed information or dense content because referrals to other specialists will be made.
3. Terminology should be used that communicates across disciplines.

4. All information should be evidence-based.

5. Information should be in formats that are familiar to physicians, such as grand rounds, algorithms, and patient education materials.

The Physician Survey showed that physicians most often accessed information related to newborn hearing screening from traditional sources, such as conferences. ${ }^{13}$ However, several systematic reviews of the research literature have shown that didactic lectures, typical at an association conference, are not effective in promoting change in physician performance and patient outcomes. ${ }^{21-23}$ Instead, active learning experiences are more effective in facilitating improvements in practice. Educational program planning must take into account the desired outcome of the educational experience. Use of methods that provide active and self-directed elements promote change in behavior, ${ }^{24}$ as well as use of multiple interventions, interactive small groups, printed materials, and graphic materials delivered in person by locally respected educators. ${ }^{25}$

The primary message from research is that effective continuing education for physicians requires that it be delivered through a variety of mechanisms. Relative to hearing loss among infants and young children, respondents to the Physician Survey indicated that the following types of materials are "very helpful": (1) laminated cards with clear protocol steps (64\%), (2) brochures to use in patient education (63\%), (3) a frequently updated Web site for physicians $(55 \%),(4)$ online continuing medical education courses (46\%), and (5) downloadable grand rounds materials (39\%). ${ }^{13}$

\section{NATIONAL INITIATIVES AND RESOURCES FOR EDUCATING PHYSICIANS ABOUT EHDI}

Several national initiatives to support professional education related to EHDI have resulted in resources that can be used by audiologists and other professionals in their outreach efforts with physicians. These are outlined in Table 1 and described in more detail in the following section. 
Boys Town National Research Hospital with the support from the National Institute of Deafness and Other Communication Disorders has developed a Web site (www. babyhearing.org) with family-friendly information that can be accessed online or downloaded and printed, specifically intended to help families through the screening, diagnosis, and intervention processes. ${ }^{18}$ Information is available in both English and Spanish, and information specifically created for healthcare providers is currently being added, making this site a comprehensive resource for all stakeholders in the EHDI process.

The AAP, in collaboration with the $\mathrm{Na}$ tional Center for Hearing Assessment and Management (NCHAM) and other organizations, has taken the initiative to reach out to its membership to educate them about newborn screening and follow-up. Each state has an AAP "chapter champion" member who takes on the responsibility of educating fellow physicians and other stakeholders through grand rounds, newsletters, and personal contact. $^{26}$ The AAP also actively disseminates information and materials specific to newborn hearing screening and the medical home. ${ }^{27,28}$ Tools that may be particularly helpful in outreach efforts with physicians include the following:

- Universal Newborn Hearing Screening, Diagnosis, and Intervention-Guidelines for Pediatric Medical Home Providers, and the accompanying patient chart checklist. ${ }^{27}$ This algorithm serves as a guide to help physicians understand the timing and components of services when an infant fails the newborn hearing screening. Some states have included this flowchart when they send newborn hearing screening referral letters to physicians. Audiologists also can print these forms and pass them on to physicians at the time of diagnosis.

- Policy statements and recent articles about topics related to hearing loss among infants and young children, such as genetics of hearing loss, mild and unilateral hearing loss, and management of auditory neuropathy. ${ }^{27}$

- PediaLink online continuing medical educational module, specific to hearing loss. ${ }^{28}$
Although there is a charge for accessing this module, pediatricians may be most comfortable accessing information from medical sources with which they are familiar.

Another information resource for healthcare providers is NCHAM at Utah State University, which serves as the national EHDI technical assistance system with funding from the Maternal and Child Health Bureau. Through collaborative partnerships, NCHAM has developed materials for a wide range of professionals engaged in EHDI efforts to ensure that infants and toddlers with hearing loss are identified as early as possible and are provided with timely and appropriate services. Many of these materials are available at www.infanthearing.org. Resources that may be particularly helpful in outreach efforts with physicians include the following:

- Contact information for state EHDI program coordinators. ${ }^{29}$ It may be useful to give this contact information to physicians to facilitate their efforts in obtaining newborn hearing screening results for their patients, as well as access to other state resources.

- Early childhood hearing screening. Objective hearing screening of infants and toddlers using otoacoustic emissions technology is being conducted successfully in a variety of early childhood settings. ${ }^{30,31}$ Multimedia training materials to help providers screen in health-care settings can be ordered or downloaded for free from www.infanthearing.org.

- Sound Beginnings video. This parent education video about newborn hearing screening can be viewed or downloaded from www.infanthearing.org. ${ }^{32}$ It is available in English and Spanish and is ideal for use in a healthcare provider's office for patient education.

\section{SUMMARY}

Over the past decade, the United States has made significant strides in reducing the age at which hearing loss is identified. Much work remains to be done to reach the goal of providing timely, comprehensive, coordinated, and effective care to all infants and young children 
with permanent hearing loss. To this end, communication and collaboration between professionals is vital to supporting families seamlessly through the EHDI process. Through educational outreach to physicians and other health-care providers, audiologists can help provide more effective services to children and families by:

1. Initiating communication with the primary care physician when a child is diagnosed with hearing loss.

2. Being familiar with local, state, and national resources for families and professionals.

3. Sharing written evidence-based information with physicians.

4. Maintaining communication with the primary care physician throughout the process, from screening through intervention.

\section{REFERENCES}

1. National Center for Hearing Assessment and Management (NCHAM). Universal newborn bearing screening: summary statistics of UNHS in the United States. Available at: http://www.infant hearing.org/status/unhsstate.html. Accessed April 15,2008

2. White KR, Mauk GW, Culpepper NB, Weirather $Y$. Newborn hearing screening in the United States: is it becoming the standard of care? In: Spivak L, ed. Universal Newborn Hearing Screening. New York, NY: Thieme Medical Publishers; 1998:225-255

3. National Center for Hearing Assessment and Management (NCHAM). Legislative activities. Available at: http://www.infanthearing.org/legislative/summary/index.html. Accessed April 15, 2008

4. Centers for Disease Control and Prevention. National EHDI Goals. Available at: http:// www.cdc.gov/NCBDD/ehdi/nationalgoals.htm. Accessed April 15, 2008

5. Centers for Disease Control and Prevention. Preliminary summary of 2006 national EHDI data (version 1). Available at: http://www.cdc.gov/ ncbddd/ehdi/documents/EHDI_Summ_2006_ Web.pdf. Accessed April 15, 2008

6. Kennedy CR, McCann DC, Campbell MJ, et al. Language ability after early detection of permanent childhood hearing impairment. $\mathrm{N}$ Engl J Med 2006;354(20):2131-2141
7. Moeller MP. Early intervention and language development in children who are deaf and hard of hearing. Pediatrics 2000;106(3):e431120-e431135

8. Yoshinaga-Itano C. From screening to early identification and intervention: discovering predictors to successful outcomes for children with significant hearing loss. J Deaf Stud Deaf Educ 2003;8(1):11-30

9. Medical Home Initiatives for Children with Special Needs Project Advisory Committee. American Academy of Pediatrics. The medical home. Pediatrics 2002;110:184-186

10. White KR. Early intervention for children with permanent hearing loss: finishing the EHDI revolution. Volta Rev 2007;106(3):237-258

11. Joint Committee on Infant Hearing. Year 2007 position statement: principles and guidelines for early hearing detection and intervention programs. Pediatrics 2007;120(4):898-921

12. Moeller MP, Eiten L, White KR, Shisler L. Strategies for educating physicians about newborn hearing screening. J Acad Rehabil Audiol 2009; in press

13. Moeller MP, White KR, Shisler L. Primary care physicians' knowledge, attitudes, and practices related to newborn hearing screening. Pediatrics 2006;118:1357-1370

14. National Center for Hearing Assessment and Management (NCHAM). Survey of EHDI Program Practices Related to Federal Privacy Regulations. Available at: http://www.infanthearing.org/ privacy/index.html. Accessed April 15, 2008

15. National Center for Hearing Assessment and Management (NCHAM). Survey of Physicians Knowledge, Attitudes, and Practices Related to Early Hearing Detection and Intervention. Available at: http://www.infanthearing.org/medical home_survey/index.html. Accessed April 15, 2008

16. ASHA. Guidelines for the Audiologic Assessment of Children from Birth to 5 Years of Age. Available at: http://www.asha.org/docs/html/ GL2004-00002.html. Accessed April 15, 2008

17. American Academy of Audiology. Pediatric amplification protocol. Available at: http://www.audio logy.org/NR/rdonlyres/53D26792-E321-41AF850F-CC253310F9DB/0/pedamp.pdf. Accessed April 15, 2008

18. Boys Town National Research Hospital. Babies hearing. Available at: http://www babyhearing.org. Accessed April 15, 2008

19. National Early Childhood Techuical Assistance Center (NECTAC). Early intervention program for infants and toddlers with disabilities (part $C$ of IDEA). Available at: http://www.nectac.org/parte/ partc.asp. Accessed April 15, 2008

20. National Center for Hearing Assessment and Management (NCHAM). Criteria for infants and toddlers with hearing loss to be eligible for early 
intervention services under IDEA. Available at: http://www.infanthearing.org/earlyintervention. Accessed April 15, 2008

21. Davis D, O'Brien MA, Freemantle N, Wolf FM, Mazmanian P, Taylor-Vaisey A. Impact of formal continuing medical education. JAMA 1999;282: 867-874

22. Davis D, Thomson M, Oxman A, Haynes B. Evidence for the effectiveness of CME: a review of 50 randomized controlled trials. JAMA 1992;268: 1111-1117

23. Thomson O'Brien MA, Freemantle N, Oxman AD, Wolf F, Davis DA, Herrin J. Continuing education meetings and workshops: effects on professional practice and health care outcomes. Cochrane Database Syst Rev 2001;CD003030:1-33

24. Mazmanian P, Davis D. Continuing medical education and the physician as a learner. Guide to the evidence. JAMA 2002;288:1057-1060

25. Cauffman J, Forsyth R, Clark V, et al. Randomized controlled trials of continuing medical education: what makes them most effective? J Contin Educ Health Prof 2002;22:214-221

26. National Center for Hearing Assessment and Management (NCHAM). American Academy of Pediatrics EHDI program information. Available at: http://www.infanthearing.org/medicalhome/ aap.html. Accessed April 15, 2008
27. American Academy of Pediatrics. Newborn and infant hearing screening activities. Available at: http:/www.medicalhomeinfo.org/screening/ hearing.html\#Tools. Accessed April 15, 2008

28. American Academy of Pediatrics. PediaLink Learning Center. Available at: http://www.pedialink.org. Accessed April 15, 2008

29. National Center for Hearing Assessment and Management (NCHAM). U.S. states, territories \& commonwealths contacts for early hearing detection \& intervention. Available at: http:// www.infanthearing.org/status/cnhs.html. Accessed April 15, 2008

30. National Center for Hearing Assessment and Management (NCHAM). Current activities: OAE screening in Head Start. Available at: http://www.infanthearing.org/earlychildhood. Accessed April 15, 2008

31. Eiserman W, Shisler L, Foust T, Buhrmann J, Winston R, White K. Screening for hearing loss in early childhood programs. Early Child Res $Q$ 2007;22(1):105-117

32. National Center for Hearing Assessment and Management (NCHAM). Educating parents about newborn hearing screening (videos). Available at: http://www.infanthearing.org/videos/ index.html\#parents. Accessed April 15, 2008 